\title{
THE THIRD AND FOURTH MOMENTS OF THE LOCAL STELLAR VELOCITY DISTRIBUTION
}

\author{
R. H. MILLER \\ University of Chicago, U.S.A.
}

\begin{abstract}
The velocity moments through the fourth order were calculated for a subset of 870 astrophysically selected stars from Gliese's catalog. An essential part of this program was the determination of the covariances of estimates of the moments; both sampling and observational error contributions were taken into account in evaluating the total covariance matrix. In applications to the study of galactic structure, the Gliese stars are regarded as tracers of the galactic potential. The moments found are consistent with conventional assumptions about symmetries of the Galaxy. Certain galactic parameters, such as the curvature in the law of rotation and the asymmetric drift, were studied with the help of the values obtained for the moments.
\end{abstract}

The higher moments of the local stellar velocity distribution can be of use in several ways in the study of the properties of the Galaxy. Three problems were addressed: (1) Can the third and fourth moments be determined at all? Contrary to the folklore, fourth order moments can be determined with a 'signal-to-noise ratio' of about 5-8. (2) Are the resulting moments consistent with the assumed symmetries of the Galaxy? Except for vertex deviation, they are. (3) What do the moments tell about the Galaxy? This work was largely carried out by a student, R. R. Erickson; his discussion of questions (2) and (3) follows a hydrodynamical development by P. O. Vandervoort. Gliese's (1969) catalog provided the data used for this study; the data were accepted uncritically. This sample of stars has widely appreciated properties; both the strengths and shortcomings of the catalog are well known. This is advantageous for a study of the kind described here in which the principal scientific contribution is the demonstration that a certain kind of problem can feasibly be attacked. The statistical methods used have been checked by this example, and shown to yield usable results.

The sample provided by Gliese's catalog was ruthlessly trimmed to obtain a subset that could be used in the required manner, but all criteria were based on data contained in the catalog. Any entry that was incomplete or uncertain was thrown out; this included any system for which radial velocity determination was based on a white dwarf. Multiple systems were treated as a single entry. Among the acceptance criteria, that most likely to be controversial was the rejection of subdwarfs and of stars listed as possible subdwarfs. This was necessary because there are too few subdwarfs to provide a statistically usable sample. The final sample contained 870 stars.

In describing the statistical method, many important details must be omitted. Only a few of the most important features will be described. The appropriate method for estimating moment-like functions is a tensor generalization of Fisher's $k$-statistic, for which a sampling theory is available (Kaplan, 1952). Error estimates are the essence of the problem since the question of whether the higher moments can be determined must be decided on the relative sizes of the moments and of their estimated errors 
(signal-to-noise ratio or coefficient of variation). The final covariance is the sum of two parts: the propagated observational errors (available from Gliese's catalog) and the sampling errors. Sampling errors are familiar as the term the yields the usual $1 / \sqrt{N}$ relative accuracy. Determination of the sampling covariances between two fourthorder moments requires the calculation of an eighth-order moment. There are 31 non-trivial moments, so the covariance matrix is $31 \times 31$ real symmetric positive definite. The full set of moments and the full covariance matrix, along with the details of the calculation, will be given elsewhere.

TABLE I

Values of the product $\Delta \mathbf{x}^{t} \mathrm{C}^{-1} \Delta \mathbf{x}=\Delta \chi^{2}$

\begin{tabular}{lll}
\hline Subsample & $\begin{array}{l}\text { Number of } \\
\text { systems }\end{array}$ & $\Delta \chi^{2}$ \\
\hline Half of sample (first, third, fifth, ... systems) & 435 & 24.6 \\
Half of sample (second, fourth, sixth, & & \\
... systems) & 435 & 26.0 \\
G0 to M9 & 718 & 10.9 \\
G0 to K9 & 466 & 20.6 \\
F5 to K9 & 556 & 13.5 \\
Parallax $<0^{\prime \prime 075}$ & 601 & 10.1 \\
Parallax $\geqslant 0.075$ & 269 & 43.8 \\
\hline
\end{tabular}

The comparisons are made to the basic sample of 870 stellar systems.

The feature of this statistical exercise that yields the most convincing demonstration of its validity is that the values obtained are quite stable when the moments are re-computed using various subsets of the basic 870-system sample. The results for some of these sectionings are shown in Table I; there the square of the 'distance' between the solution obtained from each of these subsets and that obtained from the full sample of 870 stars is shown, calculated using the covariance matrix belonging to the subset. For a random scatter of independent values, the distances should be $\chi^{2}$-distributed on $31 \mathrm{deg}$ of freedom (expectation value about 31 ). With due regard for the lack of independence, the sample seems remarkably stable under these sectionings.

The contributions of observational and of sampling error to the total covariance are of the same order of magnitude for the fourth-order moments, with the sampling term almost always larger. Thus this sample is nearly optimal for present purposes in the sense that an improvement in observational techniques, leading to a reduction of the observational errors, would not improve our knowledge of the moments significantly without a substantial increase in the number of stars in the sample; but neither would an increase in the number of stars help much without a corresponding improvement in observational techniques.

We are now ready to examine the values obtained for the moments from the standpoint of the assumed symmetries before applying them to the study of galactic structure. The stars of the Gliese catalog are regarded as tracers of the galactic 
potential in the applications. Completeness of the sample is not required - only that the sample be representative of stars that have lived long enough to explore the galactic potential. Stars that have undergone the violence of early galactic collapse would prejudice the results undesirably. The stars actually used should represent the disk population; they explore a toroidal region of the Galaxy that extends outward and inward by a kpc or so. That region contains so many stars that the population from which the sample is drawn is effectively infinite.

Under the usual galactic symmetry assumptions (rotational symmetry, symmetry about a plane, the only differential motion allowed is a differential rotation, and the system is in equilibrium), all moments that contain an odd number of $\pi$ (galactic radial) or $Z$ (normal to the galactic plane) indices should vanish. This is true within the statistical accuracy for all the estimated second, third, and fourth order moments except for two related to vertex deviation: the $\left\langle\pi \Theta_{p}\right\rangle$ and $\left\langle\pi \pi \pi \Theta_{p}\right\rangle$ moments. The fourth-order moments that should survive stand up at about 5-8 standard deviations.

The next exercise was to legislate that those moments that should vanish by symmetry are exactly zero: the solution is constrained so that those moments must be zero. There are 12 allowed nonzero moments. The subsequent results are quoted for 4 solutions: the full set of 31 moments (none forced to zero), the set of 12 moments with all forbidden moments forced to zero, a set of 13 that leaves the $\left\langle\pi \Theta_{p}\right\rangle$ moment free in addition to the allowed 12 , and a set of 14 that also leaves the $\left\langle\pi^{3} \Theta_{p}\right\rangle$ moment free. All of these are admissible solutions from a purely statistical point of view, although statistical criteria must be strained a bit to suppress the $\left\langle\pi \Theta_{p}\right\rangle$ term. Even if we could not see beyond $20 \mathrm{pc}$, we could conclude that the Galaxy has the assumed symmetries. The well-known non-normal character of the velocity distribution appears as well (fourth moments too large, implying too many stars in the high-velocity wings of the distribution).

The stage is now set to use the values of these moments to investigate properties of the Galaxy. The square of the axis ratio of the velocity ellipse, $B /(B-A)$, appears as the ratio of several combinations of moments; consistent values are obtained, as shown in Table II. These estimates are not independent; a 'best value' for the ratio is not a simple weighted mean, but rather is a single value consistent with all the covariances among the moments. This 'weighted mean' is quite close to the value 0.4 that results from the standard values for the Oort constants. In principle, the

TABLE II

Values of $B /(B-A)$ and their standard errors

\begin{tabular}{|c|c|c|c|c|}
\hline & $\begin{array}{l}\text { Set of } 31 \\
\text { moments }\end{array}$ & $\begin{array}{l}\text { Set of } 14 \\
\text { moments }\end{array}$ & $\begin{array}{l}\text { Set of } 13 \\
\text { moments }\end{array}$ & $\begin{array}{l}\text { Set of } 12 \\
\text { moments }\end{array}$ \\
\hline$\left\langle\Theta_{p^{2}}\right\rangle \mid\left\langle\pi^{2}\right\rangle$ & $0.45 \pm 0.04$ & $0.46 \pm 0.03$ & $0.45 \pm 0.03$ & $0.44 \pm 0.04$ \\
\hline$\left\langle\Theta_{p}^{4}\right\rangle /\left\langle\pi^{2} \Theta_{p}^{2}\right\rangle$ & 0.390 .06 & 0.390 .05 & 0.390 .05 & $0.39 \quad 0.06$ \\
\hline $3\left\langle\pi^{2} \Theta_{p}^{2}\right\rangle \mid\left\langle\pi^{4}\right\rangle$ & $0.66 \quad 0.12$ & $0.71 \quad 0.11$ & $0.65 \quad 0.11$ & $0.60 \quad 0.11$ \\
\hline$\left\langle\Theta_{p}^{2} Z^{2}\right\rangle \mid\left\langle\pi^{2} Z^{2}\right\rangle$ & $\begin{array}{ll}0.41 & 0.09\end{array}$ & $0.42 \quad 0.07$ & $0.41 \quad 0.08$ & $\begin{array}{ll}0.35 & 0.07\end{array}$ \\
\hline Resultant $B /(B-A)$ & 0.430 .03 & 0.410 .03 & $0.42 \quad 0.03$ & $0.42 \quad 0.03$ \\
\hline
\end{tabular}


number of independent parameters could be further reduced by eliminating 4 of these moments in favor of the ratio $B /(B-A)$ to leave a 9-parameter solution, but we have not yet done this. The hydrodynamical equations do not permit separate solutions for $B$ and $A$.

Some of the galactic parameters obtainable through these arguments are shown in Table III. There, $l_{v}$ is the longitude of the vertex, which vanishes for the 12-moment solution. The quantity $D=\partial^{2}\left(\ln \Theta_{0}\right) / \partial(\ln \varpi)^{2}$, is essentially the curvature in the law of galactic rotation. A mixture of the radial 'scale heights' for $N$, the number density, and for $\left\langle\pi^{2}\right\rangle$, the velocity dispersion in the radial direction, appears as $\Lambda$; the scale height is around $5 \mathrm{kpc}$. The two quantities that contribute to $\Lambda$ cannot be separated without additional assumptions; two 'reasonable' assumptions appear as $\partial(\ln Q) /$ $\partial \omega=0$ or 0.1 ; these assumptions concern arguments relating to the stability of the Galaxy (itself a slippery argument that need not be entered upon here). The scaleheights obtained are fairly insensitive to the value assumed. Once a value is assumed, it is possible to calculate the asymmetric drift - it is remarkable that the asymmetric drift can be determined from a sample such as this. The quantity, $\partial\left(\ln \left\langle\pi^{2}\right\rangle\right) / \partial \varpi$ is sometimes regarded as too small to admit observational determination (Oort, 1965). This cannot be so: a value of $\partial\left(\ln \left\langle\pi^{2}\right\rangle\right) / \partial w=0$ leads to values for $\partial(\ln N) / \partial w=$ $=-1.8 \pm 0.5 \mathrm{kpc}^{-1}$, or to a radial scale-height in the number density of $550 \mathrm{pc}$, a value more appropriate to vertical scale-heights.

It has not been possible, in this report, to present more than a brief synopsis of this work. It is presented with the hope of stimulating your interest by indicating the usefulness of results of this kind in the study of galactic structure. Papers containing the full details of the statistical work, the development of the equations of

TABLE III

Galactic parameters and their standard errors

\begin{tabular}{|c|c|c|c|c|c|c|c|c|}
\hline \multirow{3}{*}{$l_{v}$} & \multicolumn{2}{|c|}{$\begin{array}{l}\text { Set of } 31 \\
\text { moments }\end{array}$} & \multicolumn{2}{|c|}{$\begin{array}{l}\text { Set of } 14 \\
\text { moments }\end{array}$} & \multicolumn{2}{|c|}{$\begin{array}{l}\text { Set of } 13 \\
\text { moments }\end{array}$} & \multicolumn{2}{|c|}{$\begin{array}{l}\text { Set of } 12 \\
\text { moments }\end{array}$} \\
\hline & \multicolumn{2}{|c|}{$9^{\circ} \pm 3^{\circ}$} & \multicolumn{2}{|c|}{$9^{\circ} \pm 2^{\circ}$} & \multicolumn{2}{|c|}{$7^{\circ} \pm 2^{\circ}$} & \multicolumn{2}{|c|}{ suppressed } \\
\hline & 0.6 & 1.4 & 0.2 & 1.4 & 0.2 & 1.5 & -0.1 & 1.5 \\
\hline$\partial^{2} \Theta_{0} / \partial w^{2}\left(\mathrm{~km} \mathrm{~s}^{-1} \mathrm{kpc}^{-2}\right)$ & 2.2 & 3.6 & 1.2 & 3.4 & 1.0 & 3.6 & 0.5 & 3.7 \\
\hline$\Lambda(\mathrm{kpc})$ & 4.6 & 0.9 & 4.3 & 0.9 & 4.6 & 1.0 & 5.1 & 1.1 \\
\hline \multicolumn{9}{|l|}{ For $\partial \ln Q^{2} / \partial w=0.0 \mathrm{kpc}^{-1}$} \\
\hline$\partial \ln N / \partial \pi\left(\mathrm{kpc}^{-1}\right)$ & -0.20 & 0.07 & -0.22 & 0.07 & -0.22 & 0.07 & -0.22 & 0.07 \\
\hline$\partial \ln \left\langle\pi^{2}\right\rangle / \partial w\left(\mathrm{kpc}^{-1}\right)$ & -0.22 & 0.05 & -0.23 & 0.06 & -0.21 & 0.06 & -0.19 & 0.06 \\
\hline$\Theta_{0}-\Theta_{c}\left(\mathrm{~km} \mathrm{~s}^{-1}\right)$ & -9 & 3 & -9 & 4 & -9 & 3 & -8 & 3 \\
\hline \multicolumn{9}{|l|}{ For $\partial \ln Q^{2} / \partial \varpi=0.1 \mathrm{kpc}^{-1}$ : } \\
\hline$\partial \ln N / \partial w\left(\mathrm{kpc}^{-1}\right)$ & -0.25 & 0.07 & -0.26 & 0.07 & -0.27 & 0.07 & -0.27 & 0.07 \\
\hline$\partial \ln \left\langle\pi^{2}\right\rangle / \partial \varpi\left(\mathrm{kpc}^{-1}\right)$ & -0.21 & 0.05 & -0.23 & 0.06 & -0.21 & 0.06 & -0.19 & 0.06 \\
\hline$\Theta_{0}-\Theta_{c}\left(\mathrm{~km} \mathrm{~s}^{-1}\right)$ & -9 & 3 & -10 & 4 & -9 & 3 & -9 & 3 \\
\hline
\end{tabular}

Where needed in calculating these parameters, the 'standard' self-consistent set of values $\{A=15 \mathrm{~km}$ $\mathrm{s}^{-1}, B=10 \mathrm{~km} \mathrm{~s}^{-1}, \Theta_{0}=250 \mathrm{~km} \mathrm{~s}^{-1}$, and $\left.w=10 \mathrm{kpc}\right\}$ were used. The standard errors (equal to the probable errors divided by 0.6745 ) that are listed reflect only the propagation of the observational and sampling errors of the moments and do not include any uncertainties in the 'standard' values. 
stellar hydrodynamics to include fourth-order moments, and the application to galactic structure, have been submitted to The Astrophysical Journal by Erickson and by Vandervoort.

\section{References}

Gliese, W.: 1969, Veröffentl. Astron. Rechen-Inst. Heidelberg, No. 22.

Kaplan, E. L.: 1952, Biometrika 39, 319.

Oort, J. H.: 1965, Stars and Stellar Systems 5, 455. 